SLAC-PUB-8173

June 1999

\title{
Neutrino masses and sneutrino mixing in $R$-parity violating supersymmetry
}

\author{
Yuval Grossman ${ }^{\mathrm{a}}$ and Howard E.. Haber ${ }^{\mathrm{b}}$

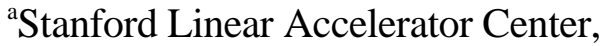 \\ Stanford University, Stanford, CA 94309 \\ ${ }^{\mathrm{b}}$ Santa Cruz Institute for Particle Physics, \\ University of California, Santa Cruz, CA 95064
}

Invited talk presented at American Physical Society (APS) Meeting of the Division of Particles and Fields (DPOF 99), 1/5/99-1/9/99, Los Angeles, CA, USA

Stanford Linear Accelerator Center, Stanford University, Stanford, CA 94309

Work supported by Department of Energy contract DE-AC03-76SF00515. 
SLAC-PUB-8173

SCIPP-99/24

hep-ph/yymmnnn

June, 1999

\title{
Neutrino masses and sneutrino mixing in R-parity violating
} supersymmetry* ${ }^{* \dagger}$

\author{
Yuval Grossman ${ }^{a}$ and Howard E. Haber ${ }^{b}$ \\ ${ }^{a}$ Stanford Linear Accelerator Center, Stanford University, Stanford, CA 94309 \\ ${ }^{b}$ Santa Cruz Institute for Particle Physics, University of California, Santa Cruz, CA 95064
}

\begin{abstract}
R-parity-violating supersymmetry with a conserved baryon number $B$ provides a framework for particle physics with lepton number $(L)$ violating interactions. Two important probes of the $L$-violating physics are neutrino masses and sneutrino-antisneutrino mass-splittings. We evaluate these quantities in the context of the most general CP-conserving, R-parity-violating $B$-conserving extension of the minimal supersymmetric standard model. In generic three-generation models, three sneutrino-antisneutrino mass splittings are generated at tree-level. In contrast, only one neutrino mass is generated at tree-level; the other two neutrinos acquire masses at one-loop. In many models, the dominant contribution to the radiative neutrino masses is induced by the non-zero sneutrino-antisneutrino mass splitting.
\end{abstract}

\footnotetext{
*Invited talk presented by Yuval Grossman at the American Physical Society (APS) meeting of the division of particles and fields (DPF99).

${ }^{\dagger}$ YG is supported by the U.S. Department of Energy under contract DE-AC03-76SF00515, and HEH is supported in part by the U.S. Department of Energy under contract DE-FG03-92ER40689.
} 


\section{INTRODUCTION}

The solar and atmospheric neutrino anomalies provide strong indications that the neutrinos are massive. In particular, the data suggest that there is near-maximal mixing between $\nu_{\mu}$ and $\nu_{\tau}$ but that their masses are hierarchically separated [1]. To accommodate this data, the Standard Model must be extended, either by introducing right-handed neutrinos or by adding Majorana neutrino mass terms that violate lepton number by two units.

In low-energy supersymmetric extensions of the Standard Model, lepton number and baryon number conservation is not automatically respected by the most general set of renormalizable interactions. However, the constraints on baryon number violation are extremely severe in order to avoid fast proton decay. If one wants to enforce lepton number and baryon number conservation in the tree-level supersymmetric theory, it is sufficient to impose one extra discrete symmetry. In the minimal supersymmetric standard model (MSSM), a multiplicative symmetry called R-parity is introduced, where the $\mathrm{R}$ quantum number of an MSSM field of spin $S$, baryon number $B$ and lepton number $L$ is given by $(-1)^{[3(B-L)+2 S]}$. By introducing this symmetry, one eliminates all dimension-four lepton number and baryon number-violating interactions. Majorana neutrino masses can be generated in an R-parityconserving extension of the MSSM involving new $\Delta L=2$ interactions through the supersymmetric see-saw mechanism $[2,3]$.

Such $\Delta L=2$ interaction have an important impact on sneutrino phenomena [3-5]. The sneutrino $(\tilde{\nu})$ and antisneutrino $(\overline{\tilde{\nu}})$, which are eigenstates of lepton number, are no longer mass eigenstates. The mass eigenstates are therefore superpositions of $\tilde{\nu}$ and $\overline{\tilde{\nu}}$, and sneutrino mixing effects can lead to a phenomenology analogous to that of $K-\bar{K}$ and $B$ $\bar{B}$ mixing. The mass splitting between the two sneutrino mass eigenstates is related to the magnitude of lepton number violation, which is typically characterized by the size of neutrino masses. (In some cases the sneutrino mass splitting may be enhanced by a factor as large as $10^{3}$ compared to the corresponding neutrino mass [3,6].) As a result, the sneutrino mass splitting is expected generally to be very small. Yet, it can be detected in many cases, if one is able to observe the lepton number oscillation [3].

The primary motivation for introducing a conserved R-parity above was to impose a conserved baryon number to avoid fast proton decay. However, this can also be achieved in low-energy supersymmetric models where $B$ is conserved but $L$ is violated (so that $\mathrm{R}$ parity is also violated). In this paper, we focus on the $B$-conserving R-parity-violating (RPV) extension of the MSSM. In such a model, neutrinos are massive [7-11] and sneutrinoantisneutrino pairs are no longer mass-degenerate [3-5]. In Section II, we introduce the most general RPV extension of the MSSM with a conserved baryon number and establish our notation. In Section III, we show how a tree-level mass for one neutrino is generated due to neutrino-neutralino mixing. In Section IV, we exhibit how tree-level mass splittings for the three sneutrino-antisneutrino pairs are generated due to sneutrino-Higgs bosons mixing. In Section V, we calculate the neutrino masses generated at one loop. In Section VI, we argue that in many models, the dominant contribution to the one-loop neutrino masses is induced by the non-zero sneutrino-antisneutrino mass splittings. A brief summary and conclusions are given in Section VII. 


\section{R-PARITY VIOLATION FORMALISM}

In RPV low-energy supersymmetry, there is no conserved quantum number that distinguishes the lepton supermultiplets $\hat{L}_{m}$ and the down-type Higgs supermultiplet $\hat{H}_{D}$. Here, $m$ is a generation label that runs from 1 to 3 . Each supermultiplet transforms as a $Y=-1$ weak doublet under the electroweak gauge group. It is therefore convenient to denote the four supermultiplets by one symbol $\hat{L}_{\alpha}(\alpha=0,1,2,3)$. The Lagrangian of the theory is fixed by the superpotential and the soft-supersymmetry-breaking terms.

The most general renormalizable superpotential respecting baryon number is given by:

$$
W=\epsilon_{i j}\left[-\mu_{\alpha} \hat{L}_{\alpha}^{i} \hat{H}_{U}^{j}+\frac{1}{2} \lambda_{\alpha \beta m} \hat{L}_{\alpha}^{i} \hat{L}_{\beta}^{j} \hat{E}_{m}+\lambda_{\alpha n m}^{\prime} \hat{L}_{\alpha}^{i} \hat{Q}_{n}^{j} \hat{D}_{m}-h_{n m} \hat{H}_{U}^{i} \hat{Q}_{n}^{j} \hat{U}_{m}\right]
$$

where $\hat{H}_{U}$ is the up-type Higgs supermultiplet, the $\hat{Q}_{n}$ are doublet quark supermultiplets, $\hat{U}_{m}\left[\hat{D}_{m}\right]$ are singlet up-type [down-type] quark supermultiplets and the $\hat{E}_{m}$ are the singlet charged lepton supermultiplets. (Our notational conventions follow those of ref. [4].) Without loss of generality, the coefficients $\lambda_{\alpha \beta m}$ are taken to be antisymmetric under the interchange of the indices $\alpha$ and $\beta$. Note that the $\mu$-term of the MSSM is now extended to an 4-component vector, $\mu_{\alpha}$. Then, the trilinear terms in the superpotential proportional to $\lambda$ and $\lambda^{\prime}$ contain lepton number violating generalizations of the down quark and charged lepton Yukawa matrices.

Next, we consider the most general set of (renormalizable) soft-supersymmetry-breaking terms. In addition to the usual soft-supersymmetry-breaking terms of the R-parityconserving MSSM, one must also add new $A$ and $B$ terms corresponding to the RPV terms of the superpotential. In addition, new RPV scalar squared-mass terms also exist. As above, we can streamline the notation by extending the definitions of the coefficients of the R-parity-conserving soft-supersymmetry-breaking terms to allow for an index of type $\alpha$ which can run from 0 to 3 (while Latin indices $m$ and $n$ run from 1 to 3 ). Explicitly,

$$
\begin{aligned}
V_{\text {soft }}= & \left(M_{\widetilde{Q}}^{2}\right)_{m n} \widetilde{Q}_{m}^{i *} \widetilde{Q}_{n}^{i}+\left(M_{\widetilde{U}}^{2}\right)_{m n} \widetilde{U}_{m}^{*} \widetilde{U}_{n}+\left(M_{\widetilde{D}}^{2}\right)_{m n} \widetilde{D}_{m}^{*} \widetilde{D}_{n} \\
& +\left(M_{\widetilde{L}}^{2}\right)_{\alpha \beta} \widetilde{L}_{\alpha}^{i *} \widetilde{L}_{\beta}^{i}+\left(M_{\widetilde{E}}^{2}\right)_{m n} \widetilde{E}_{m}^{*} \widetilde{E}_{n}+m_{U}^{2}\left|H_{U}\right|^{2}-\left(\epsilon_{i j} b_{\alpha} \widetilde{L}_{\alpha}^{i} H_{U}^{j}+\text { h.c. }\right) \\
& +\epsilon_{i j}\left[\frac{1}{2} a_{\alpha \beta m} \widetilde{L}_{\alpha}^{i} \widetilde{L}_{\beta}^{j} \widetilde{E}_{m}+a_{\alpha n m}^{\prime} \widetilde{L}_{\alpha}^{i} \widetilde{Q}_{n}^{j} \widetilde{D}_{m}-\left(a_{U}\right)_{n m} H_{U}^{i} \widetilde{Q}_{n}^{j} \widetilde{U}_{m}+\text { h.c. }\right] \\
& +\frac{1}{2}\left[M_{3} \widetilde{g} \widetilde{g}+M_{2} \widetilde{W}^{a} \widetilde{W}^{a}+M_{1} \widetilde{B} \widetilde{B}+\text { h.c. }\right] .
\end{aligned}
$$

Note that the single $B$ term of the MSSM is extended to a 4-component vector $b_{\alpha}$, and the squared-mass term for the down-type Higgs boson and the $3 \times 3$ lepton scalar squared-mass matrix are combined into a $4 \times 4$ matrix. In addition, the matrix $A$-parameters of the MSSM are extended in the obvious manner [analogous to the Yukawa coupling matrices in eq. (1)]; in particular, $a_{\alpha \beta m}$ is antisymmetric under the interchange of $\alpha$ and $\beta$. It is sometimes convenient to follow the more conventional notation in the literature and define the $A$ and $B$ parameters as follows:

$$
\begin{aligned}
& a_{\alpha \beta m} \equiv \lambda_{\alpha \beta m}\left(A_{E}\right)_{\alpha \beta m}, \quad\left(a_{U}\right)_{n m} \equiv h_{n m}\left(A_{U}\right)_{n m}, \\
& a_{\alpha n m}^{\prime} \equiv \lambda_{\alpha n m}^{\prime}\left(A_{D}\right)_{\alpha n m}, \quad b_{\alpha} \equiv \mu_{\alpha} B_{\alpha} \text {, }
\end{aligned}
$$

where repeated indices are not summed over in the above equations. Finally, the Majorana gaugino masses, $M_{i}$, are unchanged from the MSSM. 
The total scalar potential is given by:

$$
V_{\text {scalar }}=V_{F}+V_{D}+V_{\text {soft }},
$$

where explicit forms for the supersymmetric contributions $V_{F}$ and $V_{D}$ can be found in ref. [4]. We do not present here the minimization conditions, but only mention that regions of parameter space exist where only the neutral color-singlet scalar fields acquire vacuum expectation values: $\left\langle h_{U}\right\rangle \equiv v_{u} / \sqrt{2}$ and $\left\langle\tilde{\nu}_{\alpha}\right\rangle \equiv v_{\alpha} / \sqrt{2}$.

$\mathrm{Up}$ to this point, there is no preferred direction in the generalized generation space spanned by the $\hat{L}_{\alpha}$. However, It is sometime convenient to choose a particular "interaction" basis such that $v_{m}=0$, in which case $v_{0}=v_{d}$. In this basis, we denote $\hat{L}_{0} \equiv \hat{H}_{D}$.

For simplicity, we shall impose CP-invariance on the model (which implies that all parameters appearing in eqs. (1) and (2) and the vacuum expectation values of the scalar fields can be taken to be real). The consequences of CP-violating effects in this model will be considered elsewhere.

\section{NEUTRINO MASS AT TREE LEVEL}

The neutrino can become massive due to mixing with the neutralinos [7]. This is determined by the $7 \times 7$ mass matrix in a basis spanned by the two neutral gauginos $\widetilde{B}$ and $\widetilde{W}^{3}$, the higgsinos $\widetilde{h}_{U}$ and $\widetilde{h}_{D} \equiv \nu_{0}$, and 3 generations of neutrinos, $\nu_{m}$. The tree-level fermion mass matrix, with rows and columns corresponding to $\left\{\widetilde{B}, \widetilde{W}^{3}, \widetilde{h}_{U}, \nu_{\beta}\right\}$ is given by $[8,9]$ :

$$
M^{(\mathrm{n})}=\left(\begin{array}{cccc}
M_{1} & 0 & m_{Z} s_{W} v_{u} / v & -m_{Z} s_{W} v_{\beta} / v \\
0 & M_{2} & -m_{Z} c_{W} v_{u} / v & m_{Z} c_{W} v_{\beta} / v \\
m_{Z} s_{W} v_{u} / v & -m_{Z} c_{W} v_{u} / v & 0 & \mu_{\beta} \\
-m_{Z} s_{W} v_{\alpha} / v & m_{Z} c_{W} v_{\alpha} / v & \mu_{\alpha} & 0_{\alpha \beta}
\end{array}\right),
$$

where $c_{W} \equiv \cos \theta_{W}, s_{W} \equiv \sin \theta_{W}$, and $0_{\alpha \beta}$ is the $4 \times 4$ zero matrix. In a basis-independent analysis, it is convenient to introduce:

$$
\cos \xi \equiv \frac{\sum_{\alpha} v_{\alpha} \mu_{\alpha}}{v_{d} \mu}
$$

where

$$
\mu^{2} \equiv \sum_{\alpha} \mu_{\alpha}^{2}, \quad v_{d}^{2} \equiv \sum_{\alpha} v_{\alpha}^{2}, \quad v^{2}=v_{u}^{2}+v_{d}^{2} \simeq(246 \mathrm{GeV})^{2}
$$

Note that $\xi$ measures the misalignment of $v_{\alpha}$ and $\mu_{\alpha}$.

It is easy to check that $M^{(n)}$ possesses 2 zero eigenvalues. We shall identify the corresponding states with 2 physical neutrinos of the Standard Model [8], while one neutrino acquires mass through mixing. We can evaluate this mass by computing the product of the five non-zero eigenvalues of $M^{(n)}$ [denoted below by $\operatorname{det}^{\prime} M^{(\mathrm{n})}$ ]

$$
\operatorname{det}^{\prime} M^{(\mathrm{n})}=m_{Z}^{2} \mu^{2} M_{\tilde{\gamma}} \cos ^{2} \beta \sin ^{2} \xi,
$$


where $M_{\tilde{\gamma}} \equiv \cos ^{2} \theta_{W} M_{1}+\sin ^{2} \theta_{W} M_{2}$. We compare this result with the product of the four neutralino masses of the R-parity-conserving MSSM (obtained by computing the determinant of the upper $4 \times 4$ block of $M^{(n)}$ with $\mu_{0}, v_{0}$ replaced by $\mu, v_{d}$ respectively)

$$
\operatorname{det} M_{0}^{(\mathrm{n})}=\mu\left(m_{Z}^{2} M_{\tilde{\gamma}} \sin 2 \beta-M_{1} M_{2} \mu\right) \text {. }
$$

To first order in the neutrino mass, the neutralino masses are unchanged by the R-parity violating terms, and we end up with [9]

$$
m_{\nu}=\frac{\operatorname{det}^{\prime} M^{(\mathrm{n})}}{\operatorname{det} M_{0}^{(\mathrm{n})}}=\frac{m_{Z}^{2} \mu M_{\tilde{\gamma}} \cos ^{2} \beta \sin ^{2} \xi}{m_{Z}^{2} M_{\tilde{\gamma}} \sin 2 \beta-M_{1} M_{2} \mu} .
$$

Thus, $m_{\nu} \sim m_{Z} \cos ^{2} \beta \sin ^{2} \xi$, assuming that all the relevant masses are at the electroweak scale.

A necessary and sufficient condition for $m_{\nu} \neq 0$ (at tree-level) is $\sin \xi \neq 0$, which implies that $\mu_{\alpha}$ and $v_{\alpha}$ are not aligned. This is generic in RPV models. In particular, the alignment of $\mu_{\alpha}$ and $v_{\alpha}$ is not renormalization group invariant $[9,10]$. Thus, exact alignment at the low-energy scale can only be implemented by the fine-tuning of the model parameters.

\section{SNEUTRINO MASS SPLITTING}

In RPV low-energy supersymmetry, the sneutrinos mix with the Higgs bosons. Under the assumption of CP-conservation, we may separately consider the CP-even and CP-odd scalar sectors. Consider first the case of one sneutrino generation. If R-parity is conserved, the CP-even scalar sector consists of two Higgs scalars $\left(h^{0}\right.$ and $H^{0}$, with $\left.m_{h^{0}}<m_{H^{0}}\right)$ and $\tilde{\nu}_{+}$, while the CP-odd scalar sector consists of the Higgs scalar, $A^{0}$, the Goldstone boson (which is absorbed by the $Z$ ), and one sneutrino, $\tilde{\nu}_{-}$. Moreover, the $\tilde{\nu}_{ \pm}$are mass degenerate, so that the standard practice is to define eigenstates of lepton number: $\widetilde{\nu} \equiv\left(\tilde{\nu}_{+}+i \tilde{\nu}_{-}\right) / \sqrt{2}$ and $\overline{\tilde{\nu}} \equiv \tilde{\nu}^{*}$. When R-parity is violated, the sneutrinos in each CP-sector mix with the corresponding Higgs scalars, and the mass degeneracy of $\tilde{\nu}_{+}$and $\tilde{\nu}_{-}$is broken. We expect the RPV-interactions to be small; thus, we can evaluate the resulting sneutrino mass splitting in perturbation theory.

The derivation of the CP-even and CP-odd scalar squared-mass matrices can be found in ref. [4]. Working in the $v_{m}=0$ basis and for one generation we find

$$
M_{\text {even }}^{2}=\left(\begin{array}{ccc}
b_{0} \cot \beta+\frac{1}{4}\left(g^{2}+g^{\prime 2}\right) v_{u}^{2} & -b_{0}-\frac{1}{4}\left(g^{2}+g^{\prime 2}\right) v_{u} v_{d} & -b_{1} \\
-b_{0}-\frac{1}{4}\left(g^{2}+g^{\prime 2}\right) v_{u} v_{d} & b_{0} \tan \beta+\frac{1}{4}\left(g^{2}+g^{\prime 2}\right) v_{d}^{2} & b_{1} \tan \beta \\
-b_{1} & b_{1} \tan \beta & m_{\tilde{\nu} \tilde{\nu}^{*}}^{2}
\end{array}\right),
$$

and

$$
M_{\text {odd }}^{2}=\left(\begin{array}{ccc}
b_{0} \cot \beta & b_{0} & b_{1} \\
b_{0} & b_{0} \tan \beta & b_{1} \tan \beta \\
b_{1} & b_{1} \tan \beta & m_{\tilde{\nu} \tilde{\nu}^{*}}^{2}
\end{array}\right)
$$


where

$$
m_{\tilde{\nu} \tilde{\nu}^{*}}^{2} \equiv\left(M_{\tilde{L}}^{2}\right)_{11}+\mu_{1}^{2}-\frac{1}{8}\left(g^{2}+g^{\prime 2}\right)\left(v_{u}^{2}-v_{d}^{2}\right) .
$$

In the R-parity-conserving limit $\left(b_{1}=\mu_{1}=0\right)$, one obtains the usual MSSM tree-level masses for the Higgs bosons and the sneutrinos.

In both squared-mass matrices [eqs. (11) and (12)], $b_{1} \ll m_{Z}^{2}$ is a small parameter that can be treated perturbatively. We may then compute the sneutrino mass splitting due to the small mixing with the Higgs bosons. Using second order matrix perturbation theory to compute the eigenvalues, we find:

$$
\begin{aligned}
& m_{\tilde{\nu}_{+}}^{2}=m_{\tilde{\nu} \tilde{\nu}^{*}}^{2}+\frac{b_{1}^{2}}{\cos ^{2} \beta}\left[\frac{\sin ^{2}(\beta-\alpha)}{\left(m_{\tilde{\nu} \tilde{\nu}^{*}}^{2}-m_{H^{0}}^{2}\right)}+\frac{\cos ^{2}(\beta-\alpha)}{\left(m_{\tilde{\nu} \tilde{\nu}^{*}}^{2}-m_{h^{0}}^{2}\right)}\right] \\
& m_{\tilde{\nu}_{-}}^{2}=m_{\tilde{\nu} \tilde{\nu}^{*}}^{2}+\frac{b_{1}^{2}}{\left(m_{\tilde{\nu} \tilde{\nu}^{*}}^{2}-m_{A^{0}}^{2}\right) \cos ^{2} \beta} .
\end{aligned}
$$

Above, we employ the standard notation for the MSSM Higgs sector observables [12]. Note that at leading order in $b_{1}^{2}$, it suffices to use the values for the Higgs parameters in the Rparity-conserving limit. Then at leading order in $b_{1}^{2}$ for the sneutrino squared-mass splitting, $\Delta m_{\tilde{\nu}}^{2} \equiv m_{\tilde{\nu}_{+}}^{2}-m_{\tilde{\nu}_{-}}^{2}$ we find

$$
\Delta m_{\tilde{\nu}}=\frac{2 b_{1}^{2} m_{Z}^{2} m_{\tilde{\nu} \tilde{\nu}^{*}} \sin ^{2} \beta}{\left(m_{\tilde{\nu} \tilde{\nu}^{*}}^{2}-m_{H}^{2}\right)\left(m_{\tilde{\nu} \tilde{\nu}^{*}}^{2}-m_{h}^{2}\right)\left(m_{\tilde{\nu} \tilde{\nu}^{*}}^{2}-m_{A}^{2}\right)} .
$$

where $\Delta m_{\tilde{\nu}}^{2} \simeq 2 m_{\tilde{\nu} \tilde{\nu}^{*}} \Delta m_{\tilde{\nu}}$.

We now extend the above results to more than one generation of sneutrinos. In a basis where $v_{m}=0$, the resulting $\mathrm{CP}$-even and CP-odd squared mass matrices are obtained from eqs. (11) and (12) by replacing $b_{1}$ with the three-dimensional vector $b_{m}$ and $m_{\tilde{\nu} \tilde{\nu}^{*}}^{2}$ by the $3 \times 3$ matrix

$$
\left(M_{\tilde{\nu} \tilde{\nu}^{*}}^{2}\right)_{m n} \equiv\left(M_{\tilde{L}}^{2}\right)_{m n}+\mu_{m} \mu_{n}-\frac{1}{8}\left(g^{2}+g^{\prime 2}\right)\left(v_{u}^{2}-v_{d}^{2}\right) \delta_{m n} .
$$

In general, $\left(M_{\tilde{\nu} \tilde{\nu}^{*}}^{2}\right)_{m n}$ is not expected to be flavor diagonal. In this case, the theory would predict sneutrino flavor mixing in addition to the sneutrino-antisneutrino mixing exhibited above. The relative strength of these effects depends on the relative size of the RPV and flavor-violating parameters of the model. To analyze the resulting sneutrino spectrum, we choose a basis in which squared-mass matrix $\left(M_{\tilde{\nu} \tilde{\nu}^{*}}^{2}\right)_{m n}=\left(m_{\tilde{\nu} \tilde{\nu}^{*}}^{2}\right)_{m} \delta_{m n}$ is diagonal. In this basis $b_{m}$ is also suitably redefined. (We will continue to use the same symbols for these quantities in the new basis.) The CP-even and CP-odd sneutrino mass eigenstates will be denoted by $\left(\tilde{\nu}_{+}\right)_{m}$ and $\left(\tilde{\nu}_{-}\right)_{m}$ respectively. It is a simple matter to extend the perturbative analysis of the scalar squared-mass matrices if the $\left(m_{\tilde{\nu} \tilde{\nu}^{*}}^{2}\right)_{m}$ are non-degenerate. We then find that $\left(\Delta m_{\tilde{\nu}}^{2}\right)_{m} \equiv\left(m_{\tilde{\nu}_{+}}^{2}\right)_{m}-\left(m_{\tilde{\nu}_{-}}^{2}\right)_{m}$ is given by eq. (15), with the replacement of $b_{1}$ and $m_{\tilde{\nu} \tilde{\nu}^{*}}^{2}$ by $b_{m}$ and $\left(m_{\tilde{\nu} \tilde{\nu}^{*}}^{2}\right)_{m}$, respectively:

$$
\left(\Delta m_{\tilde{\nu}}\right)_{m}=\frac{2 b_{m}^{2} m_{Z}^{2}\left(m_{\tilde{\nu} \tilde{\nu}^{*}}\right)_{m} \sin ^{2} \beta}{\left(\left(m_{\tilde{\nu} \tilde{\nu}^{*}}^{2}\right)_{m}-m_{H}^{2}\right)\left(\left(m_{\tilde{\nu} \tilde{\nu}^{*}}^{2}\right)_{m}-m_{h}^{2}\right)\left(\left(m_{\tilde{\nu} \tilde{\nu}^{*}}^{2}\right)_{m}-m_{A}^{2}\right)} .
$$

Since eq. (17) has been derived in the $v_{m}=0$ basis, it follows that in an arbitrary basis, all sneutrino-antisneutrino pairs would be mass-degenerate if $b_{\alpha}$ and $v_{\alpha}$ were aligned. However, this alignment is not renormalization-group invariant. Hence we expect that all the sneutrino-antisneutrino pairs are generically split in mass at tree-level. 


\section{ONE-LOOP NEUTRINO MASSES}

In contrast to the sneutrino sector, only one neutrino mass is generated at tree-level due to neutrino mixing with the neutralinos. Masses for the remaining massless neutrinos will be generated by one loop effects. There are two classes of one loop diagrams. The first consists of fermion-sfermion loops and depends on the RPV trilinear terms. The second, which in many cases is the dominant one, consists of sneutrino-neutralino loops and depends on the sneutrino-antisneutrino mass splitting. We now discuss both of these effects in turn.

First, consider the fermion-sfermion loops. Contributions to the neutrino mass matrix are generated from diagrams involving a charged lepton-slepton loop and an analogous downtype quark-squark loop [7]. In the limit where the fermion masses can be neglected,

$$
\left(m_{\nu}\right)_{q m}=\frac{1}{32 \pi^{2}}\left[\sum_{\ell, p} \lambda_{q \ell p} \lambda_{m p \ell} m_{\ell} \sin 2 \phi_{\ell} \ln \left(\frac{M_{p_{1}}^{2}}{M_{p_{2}}^{2}}\right)+3 \sum_{d, r} \lambda_{q d r}^{\prime} \lambda_{m r d}^{\prime} m_{d} \sin 2 \phi_{d} \ln \left(\frac{M_{r_{1}}^{2}}{M_{r_{2}}^{2}}\right)\right]
$$

where $\phi_{\ell}\left(\phi_{d}\right)$ is the mixing angle of the charge slepton (down type squark) squared-mass matrix,

$$
\sin 2 \phi_{\ell}=\frac{2 A_{\ell} m_{\ell}}{\sqrt{\left(L^{2}-R^{2}\right)^{2}+4 A_{\ell}^{2} m_{\ell}^{2}}}
$$

Here, $A_{\ell} \equiv\left(A_{E}\right)_{0 \ell \ell}-\mu_{0} \tan \beta, L^{2} \equiv\left(M_{\widetilde{L}}^{2}\right)_{\ell \ell}+\left(T_{3}-e \sin ^{2} \theta_{W}\right) m_{Z}^{2} \cos 2 \beta$ and $R^{2} \equiv\left(M_{\widetilde{E}}^{2}\right)_{\ell \ell}+$ $\left(e \sin ^{2} \theta_{W}\right) m_{Z}^{2} \cos 2 \beta$, with $T_{3}=-1 / 2$ and $e=-1$. For $\sin 2 \phi_{d}$, take $e=-1 / 3$ and replace $M_{\widetilde{E}}^{2} \rightarrow M_{\widetilde{D}}^{2}, M_{\widetilde{L}}^{2} \rightarrow M_{\widetilde{Q}}^{2}$ and $\ell \rightarrow d$ in the above formulae.

Second, consider the sneutrino induced masses. In general, the existence of a sneutrinoantisneutrino mass splitting, which is a result of a $\Delta L=2$ interaction, generates a one-loop contribution to the neutrino mass. We have computed exactly the one-loop contribution to the neutrino mass $\left[m_{\nu}^{(1)}\right]$ from neutralino/sneutrino loops [3]. In the limit of $m_{\nu}, \Delta m_{\tilde{\nu}} \ll m_{\tilde{\nu}}$, the formulae simplify, and we find in the one generation case

$$
m_{\nu}^{(1)}=\frac{g^{2} \Delta m_{\tilde{\nu}}}{32 \pi^{2} \cos ^{2} \theta_{W}} \sum_{j} f\left(y_{j}\right)\left|Z_{j Z}\right|^{2}
$$

where $f\left(y_{j}\right)=\sqrt{y_{j}}\left[y_{j}-1-\ln \left(y_{j}\right)\right] /\left(1-y_{j}\right)^{2}$, with $y_{j} \equiv m_{\tilde{\nu}}^{2} / m_{\tilde{\chi}_{j}^{0}}^{2}$, and $Z_{j Z} \equiv Z_{j 2} \cos \theta_{W}-$ $Z_{j 1} \sin \theta_{W}$ is the neutralino mixing matrix element that projects out the $\widetilde{Z}$ eigenstate from the $j$ th neutralino. One can check that $f\left(y_{j}\right)<0.566$, and for typical values of $y_{j}$ between 0.1 and $10, f\left(y_{j}\right)>0.25$. Since $Z$ is a unitary matrix, we expect as a rough order of magnitude estimate

$$
m_{\nu} \sim 10^{-3} \Delta m_{\tilde{\nu}}
$$

In the three-generation model, a similar estimate holds for the loop contribution to each neutrino mass. 


\section{THE NEUTRINO SPECTRUM}

The neutrino spectrum is determined by the relative size of the different RPV couplings that control the three sources of neutrino masses. In the $v_{m}=0$ basis these are $\mu_{m}$ [for the tree level mass, eq. (10)], $b_{m}$ [for the sneutrino induced one loop masses, eqs. (21) and (17)] and $\lambda_{i j k}$ and $\lambda_{i j k}^{\prime}$ [for the trilinear RPV induced one loop masses, eq. (18)]. Therefore, in order to understand the structure of the neutrino spectrum we must have a framework that predicts the magnitude of these parameters. Here we give one example: models based on an abelian horizontal symmetry [13]. Further details and examples will be given in ref. [14].

Consider a simple model based on a $U(1)$ abelian horizontal symmetry, which is described in detail in ref. [13]. For our purposes, it is sufficient to mention that the order of magnitude of all the model parameters are determined by the assigned horizontal charges to the various fields in the theory. Moreover, many ratios are predicted independently of the specific charge assignment. For the parameters relevant for neutrino masses we find the following ratios

$$
\frac{b_{i}}{v \mu_{i}} \sim 1, \quad \frac{\lambda_{i j k} v}{\mu_{i}} \sim \frac{m_{j k}^{\ell}}{v}, \quad \frac{\lambda_{i j k}^{\prime} v}{\mu_{i}} \sim \frac{m_{j k}^{d}}{v},
$$

where $m_{j k}^{\ell}\left(m_{j k}^{d}\right)$ is the charged lepton (down-type quark) mass matrix. Inserting the above order of magnitude predictions into eqs. (10), (21), (17) and (18) we find that the tree level yields the dominant contribution to the heaviest neutrino mass. The two lighter neutrinos get their masses mainly from the sneutrino-neutralino loops (as a consequence of the non-zero tree-level sneutrino-antisneutrino mass splitting); these will be referred to as the sneutrino-induced masses. The trilinear RPV induced masses are suppressed compared to the sneutrino-induced masses by at least a factor of order $30 m_{b}^{4} / v^{4} \sim 10^{-6}$ and are therefore completely negligible. Moreover, the following relations hold

$$
\frac{m_{i}}{m_{3}} \sim 10^{-3} \sin ^{2} \theta_{i 3}, \quad \frac{m_{1}}{m_{2}} \sim \sin ^{2} \theta_{12} .
$$

The fact that the sneutrino-induced neutrino mass is the dominant radiative effect is not unique to the above example. For example, in models with high energy alignment, we also find that these contributions are larger than the one-loop contribution from fermionsfermion loops [14].

\section{SUMMARY AND CONCLUSIONS}

Recent experimental signals of neutrino masses and mixing may provide the first glimpse of the lepton-number violating world. In R-parity violating supersymmetric models that incorporate lepton number violation, the sneutrino-antisneutrino mass spectrum may provide additional insight to help us unravel the mystery of neutrino masses and mixing. Such models generally predict a non-trivial neutrino spectrum in which there are several sources for neutrino masses. One neutrino acquires mass at tree level via neutrino-neutralino mixing. The other two neutrinos acquire radiative masses at one loop. In many models, the dominant contribution of the radiative neutrino masses is induced by the non-zero sneutrinoantisneutrino mass splitting. A detailed study of sneutrino phenomenology at future colliders can complement the present day study of neutrino mass and mixing in order to shed light on the nature of the underlying lepton-number violation. 


\section{REFERENCES}

[1] Y. Fukuda et al. [Super-Kamiokande Collaboration], Phys. Lett. B436, 33 (1998); Phys. Rev. Lett. 81, 1562 (1998). For a recent review of the evidence for neutrino mass, see B. Kayser, hep-ph/9810513. A recent compilation of experimental evidence for neutrino mass can be found in C. Caso et al. [Particle Data Group], Eur. Phys. J. C3, 1 (1998).

[2] J. Hisano, T. Moroi, K. Tobe, M. Yamaguchi, and T. Yanagida, Phys. Lett. B357, 579 (1995); J. Hisano, T. Moroi, K. Tobe, and M. Yamaguchi, Phys. Rev. D53, 2442 (1996).

[3] Y. Grossman and H.E. Haber, Phys. Rev. Lett. 78, 3438 (1997).

[4] Y. Grossman and H.E. Haber, Phys. Rev. D59, 093008 (1999).

[5] M. Hirsch, H.V. Klapdor-Kleingrothaus and S.G. Kovalenko, Phys. Lett. B398, 311 (1997); Phys. Rev. D57, 1947 (1998); M. Hirsch, H.V. Klapdor-Kleingrothaus, S. Kolb and S.G. Kovalenko, Phys. Rev. D57, 2020 (1998).

[6] L.J. Hall, T. Moroi and H. Murayama, Phys. Lett. B424, 305 (1998).

[7] C. Aulakh and R. Mohapatra, Phys. Lett. B119, 136 (1983); L.J. Hall and M. Suzuki, Nucl. Phys. B231, 419 (1984); L.-H. Lee, Phys. Lett. B138, 121 (1984); Nucl. Phys. B246 120, (1984); G.G. Ross and J.W.F. Valle, Phys. Lett. B151, 375 (1985); J. Ellis et al., Phys. Lett. B150, 142 (1985); S. Dawson, Nucl. Phys. B261, 297 (1985); A. Santamaria and J.W.F. Valle, Phys. Lett. B195 423 (1987); K.S. Babu and R.N. Mohapatra, Phys. Rev. Lett. 64, 1705 (1990); R. Barbieri, M.M. Guzzo, A. Masiero, and D. Tommasini, Phys. Lett. B252, 251 (1990); E. Roulet and D. Tommasini, Phys. Lett. B256, 281 (1991); K. Enkvist, A. Masiero, and A. Riotto, Nucl. Phys. B373, 95 (1992); J.C. Romao and J.W.F. Valle, Nucl. Phys. B381, 87 (1992); R.M. Godbole, P. Roy, and X. Tata, Nucl. Phys. B401, 67 (1993); A.S. Joshipura and M. Nowakowski, Phys. Rev. D51, 2421 (1995); Phys. Rev. D51, 5271 (1995); F.M. Borzumati, Y. Grossman, E. Nardi and Y. Nir, Phys. Lett. B384, 123 (1996); M. Nowakowski and A. Pilaftsis, Nucl. Phys. B461, 19 (1996); H.-P. Nilles and N. Polonsky, Nucl. Phys. B484, 33 (1997).

[8] T. Banks, Y. Grossman, E. Nardi and Y. Nir, Phys. Rev. D52, 5319 (1995).

[9] E. Nardi, Phys. Rev. D55, 5772 (1997).

[10] R. Hempfling, Nucl. Phys. B478, 3 (1996); B. de Carlos and P.L. White, Phys. Rev. D54, 3427 (1996).

[11] J. Ferrandis, Valencia preprint IFIC/98-78 (1998) [hep-ph/9810371].

[12] J.F. Gunion, H.E. Haber, G. Kane and S. Dawson, The Higgs Hunter's Guide, (AddisonWesley Publishing Company, Redwood City, CA, 1990).

[13] M. Leurer, Y. Nir, and N. Seiberg, Nucl. Phys. B398, 319 (1993); Y. Nir and N. Seiberg, Phys. Lett. B309, 337 (1993); M. Leurer, Y. Nir, and N. Seiberg, Nucl. Phys. B420, 468 (1994); Y. Grossman and Y. Nir, Nucl. Phys. B448, 30 (1995).

[14] Y. Grossman and H.E. Haber, preprint in preparation. 